\title{
An Iterative Method for Single and Vertically Stacked Semiconductor Quantum Dots Simulation
}

\author{
YIMING LI \\ Department of Communication Engineering \\ and \\ Microelectronics and Information Systems Research Center \\ National Chiao Tung University \\ Hsinchu 300, Taiwan \\ ymli@faculty.nctu.edu.tw
}

\begin{abstract}
We present in this paper a computational effective nonlinear iterative method for calculating the electron energy spectra in single and vertically stacked InAs/GaAs semiconductor quantum dots. The physical model problem is formulated with the effective one electronic band Hamiltonian, the energy- and position-dependent electron effective mass approximation, and the Ben Daniel-Duke boundary conditions. The multishift QR algorithm is implemented in the nonlinear iterative method for solving the corresponding nonlinear eigenvalue problem. This method converges monotonically when solving the nonlinear Schrödinger equation for all quantum dot simulations. Numerical results show that the electron energy spectra are significantly dependent on the number of coupled layers. For the excited states, the layer dependence effect has been found to be weaker than that for the ground state. (C) 2005 Elsevier Ltd. All rights reserved.
\end{abstract}

Keywords - Single quantum dot, Vertically stacked quantum dot, Semiconductor nanostructure, Modelling and simulation, Nonlinear eigenvalue problem, Multishift QR method, Energy spectra, Electronic structure, Wave function, Coupling effect.

\section{INTRODUCTION}

Experimental fabrications and theoretical study of nanoscale semiconductor quantum dots (QDs) have been of great interest in recent years [1-14]. With the advanced nanofabrication technology, it is possible to consider another degree of freedom along the growth direction ( $z$ direction) for a set of vertically coupled QDs. One of evident features in this system is the effects of dot-to-dot interactions on the electronic structure, the electronic entanglement, and charge transfer $[5,6]$. In the theoretical modeling and numerical simulation of semiconductor QDs, most of reported works have only considered a two-dimensional (2-D) lateral geometry and confinement potential models. These works focused on a system of vertically coupled two-layers quantum dots [5-8].

Correspondence address: P.O. Box 25-178, Hsinchu 300, Taiwan.

This work is supported in part by the National Science Council (NSC) of Taiwan under Contract NSC-93-2215E-492-008, Contract NSC-94-2215-E-492-005, and Contract NSC-94-2752-E-009-003-PAE, and by the Ministry of Economic Affairs, Taiwan under Contract 93-EC-17-A-07-S1-0011. 
To thoroughly clarify the electronic structure and tunneling ability for diverse applications to memory devices, photonic devices, and optoelectronics, it will become more interest if a system of vertically stacked $n$-layers $(n \geq 1)$ QDs can be investigated with a full 3 -D approach.

In this paper, we calculate the electron energy states for a system of vertically stacked $n$-layers QDs by considering a unified 3-D model. The model is formulated with the effective one electronic band Hamiltonian, the energy- and position-dependent electron effective mass approximation, the hard-wall confinement potential, and the Ben Daniel-Duke boundary conditions. The model defined in the single and $n$-layers QDs results in a nonlinear Schrödinger equation (effective mass is a nonlinear function of energy) to be solved. Therefore, the analytical solution of this equation becomes complicated. Based on a nonlinear iterative method, the nonlinear Schrödinger equation is solved iteratively to simulate an electron confined by InAs QDs embedded in GaAs semiconductor matrix. In the numerical solution of this nonlinear Schrödinger equation, we discretize it with finite box method (the so-called finite-volume method), first. The discretized nonlincar Schrödinger equation leads to a nonlinear eigenvalue problem (the matrix of the problem is eigenvalue-dependent). Starting from an arbitrary eigenvalue, the multishift QR algorithm [15] is implemented in the nonlinear iterative method for solving the corresponding nonlinear eigenvalue problem. It is terminated when the specified eigenvalue is converged. Otherwise, the newer energy will be updated and iterations will be performed. This method allows us to solve the energy states of QDs with very cost effective computation. Due to the monotone property of the electron effective mass, this nonlinear iterative method converges for the single and vertically stacked $n$-layers QDs. Numerical results show that the QDs' transition energy is dominated by the number of stacked layers. For the excited states, the layer dependence effect has been found to be weak than that for the ground state. The distance $d$ among the layers of QDs also plays a crucial role in the tunable states of the dots. For $d=0.5 \mathrm{~nm}$, there is about $30 \%$ variation in ground state energy. This numerical investigation is constructive in studying the magneto-optical phenomena and quantum optical structures for the vertically stacked $n$-layers QDs.

This paper is organized as follows. Section 2 states the 3-D QDs model. Section 3 discusses the computational method. Section 4 shows the computed results and presents the corresponding discussions. Section 5 draws conclusions and suggestions some future works.

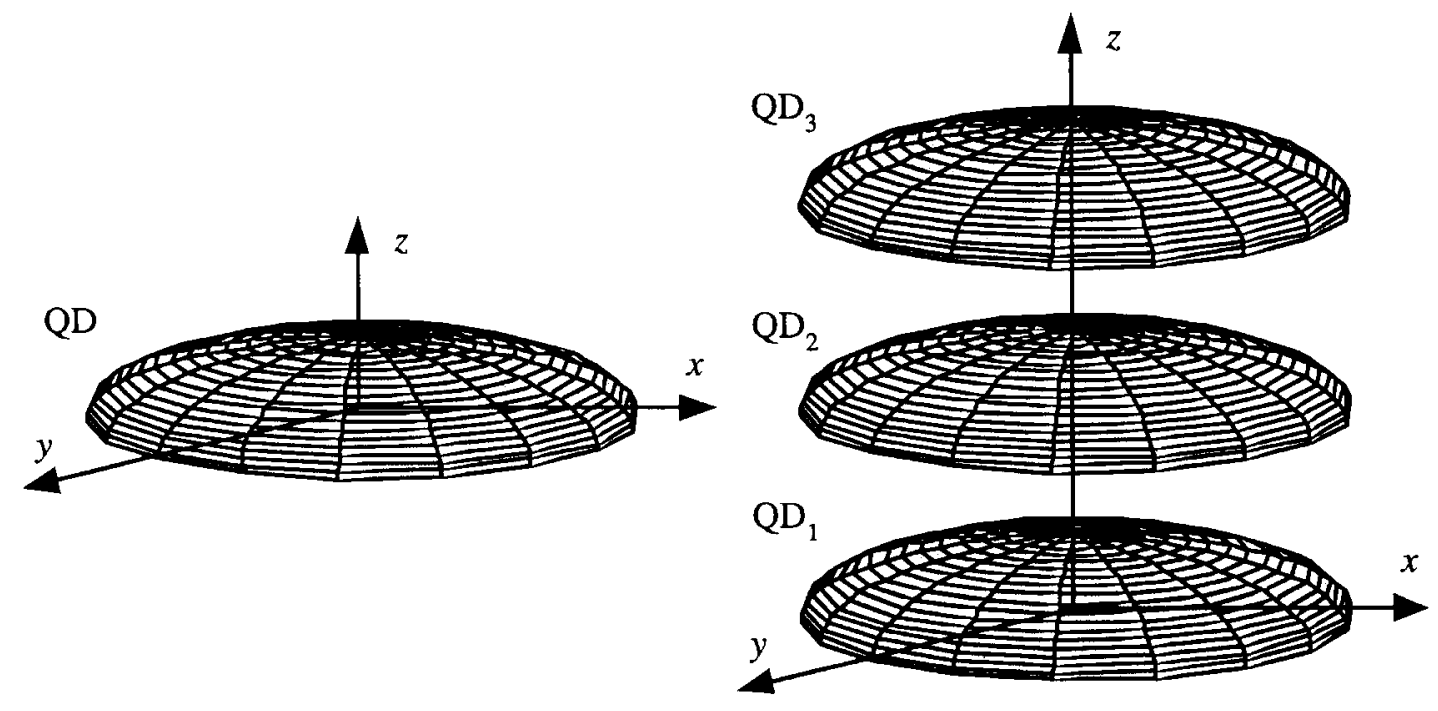

Figure 1. The left figure is A single InAs QD embedded in the GaAs semiconductor matrix. The right one shows the system of the vertically stacked three-layers InAs/GaAs QDs. 


\section{MATHEMATICAL MODEL AND COMPUTATIONAL METHOD}

As shown in Figure 1, we consider electrons confined in a system of single or vertically stacked $n$-layers QDs and apply the one-band effective Hamiltonian $H$ [9-12], we have

$$
H=-\frac{\hbar^{2}}{2} \nabla_{r}\left(\frac{1}{m(E, r)}\right) \nabla_{r}+V(r)
$$

where $m(E, r)$ is the energy- and position-dependent electron effective mass,

$$
\frac{1}{m(E, r)}=\frac{P^{2}}{\hbar^{2}}\left[\frac{2}{E+E_{g}(r)-E_{c}(r)}+\frac{1}{E+E_{g}(r)+\Delta(r)-E_{c}(r)}\right],
$$

and $V(r)=E_{c}(r)$ is the QDs' confinement potential. The $E_{c}(r), E_{g}(r), \Delta(r)$, and $P$ are the position-dependent electron band edge, band gap, spin-orbit splitting in the valance band, and momentum matrix element, respectively [9-12]. Because the QDs have ellipsoid shape with radius $R_{0}$ and of height $z_{0}$, we can treat the problem in the cylindrical coordinate $(R, \phi, z)$. The property of QDs system is cylindrical symmetry, so the wave function can be expressed as

$$
\Phi(r)=\Phi(R, z) \exp (i l \phi)
$$

where $l=0, \pm 1, \pm 2, \ldots$, is the electron orbital quantum number. Therefore, the model is

$$
-\frac{\hbar^{2}}{2 m_{i}(E)}\left(\frac{\partial^{2}}{\partial R^{2}}+\frac{\partial}{R \partial R}+\frac{\partial^{2}}{\partial z^{2}}-\frac{l^{2}}{R^{2}}\right) \Phi_{i}(R, z)+V_{i}(R, z) \Phi_{i}(R, z)=E \Phi_{i}(R, z),
$$

where $V_{i=1}(R, z)=0$ inside the dots and $V_{i=2}(R, z)=V_{0}$ outside the dots. The interface conditions (the so-called Ben Daniel-Duke boundary conditions) between two different materials are $\Phi_{1}=\Phi_{2}$ and

$$
\frac{1}{m_{1}(E)}\left(\frac{\partial \Phi_{1}}{\partial R}+\frac{d f_{s}}{d R} \frac{\partial \Phi_{1}}{\partial z}\right)=\frac{1}{m_{2}(E)}\left(\frac{\partial \Phi_{2}}{\partial R}+\frac{d f_{s}}{d R} \frac{\partial \Phi_{2}}{\partial z}\right)
$$

where $z=f_{s}(R, z)$ ( $s$ represents the ellipsoid shape QDs) is a contour generator of the QDs structure in $(R, z)$ plane. Figure 1 simply shows the single and three-layers QDs. The electron wave function vanishes at infinite physically.

As shown in Figure 2, the nonlinear Schrödinger equation is solved iteratively starting with a given initial energy $E_{0}$ to compute the final convergent energy spectra. For a given orbital quantum number, and a specified stopping criterion on energy $E$, the nonlinear iterative method consists of the following steps:

(1) set an initial energy $E_{0}$ in the interval $\left[0, V_{0}\right]$;

(2) identify the geometry of nanostructures;

(3) compute the effective mass $m$ with equation (2);

(4) solve the nonlinear Schrödinger equation as well as boundary conditions with equations $(4),(5)$;

(5) perform the convergence test on the computed newer energy $E$;

(6) if $E$ converges, then exit the iteration loops; and

(7) update the newer $E$ and back to Step 3.

This computational procedure has been proposed for single semiconductor quantum dot and quantum ring simulation in our recent works [9-12]. It is known that in the calculation of eigenvalues, the shifted- and balanced-QR is robust and converges with arbitrary initial guesses. The solution accuracy of implicitly restarted Arnoldi method relies on the initial guess of eigenvalue 


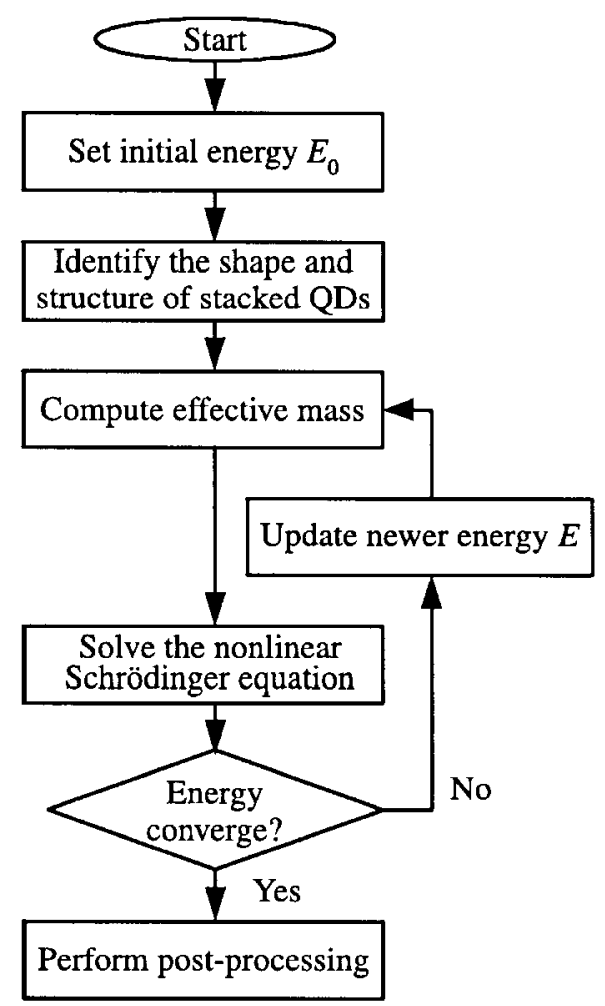

Figure 2. Simulation flowchart of the proposed computational method.

[16-18]. Any accurate calculations of electronic structure for semiconductor quantum dots depend on the discretization of Schrödinger equation over meshes. The discretization of Schrödinger equation on a fine mesh results in a large-scaled algebraic eigenvalue problem and increases the computational difficulty of the solution process. The calculation of energy states for InAs/GaAs quantum dot requires huge amount of CPU time on solving eigenvalues in Step 5. Therefore, the nonlinear iterative method is further improved in this paper by using a novel scheme, multishift QR method [15], in the solution of nonlinear algebraic eigenvalue problem of Step 5. In the numerical solution of Schrödinger equation, first, the equation is discretized with a finitebox method (the so-called finite volume method) [19]. Due to the energy-dependent of electron effective mass, the discretized nonlinear Schrödinger equation together with the corresponding Ben Daniel-Duke boundary conditions leads to a nonlinear algebraic eigenvalue problem. It is solved by the nonlinear iterative method together with the multishift QR algorithm in Step 5. The key idea of the multishift QR method is to introduce carefully chosen perturbations to reveal deflations that are not yet evident on the subdiagonal. In our experience, the proposed nonlinear iterative method converges monotonically. The cost of simulation time can be reduced about 1.5 orders of magnitude.

This solution methodology enables us to solve a large-scaled eigenvalue problem that derived from the discretization of Schrödinger equation efficiently. Once a final convergent eigenvalue is computed, the corresponding eigenvector is calculated with the inverse iteration method [20], and the post-processing is followed up. The improved nonlinear iterative method in solving the nonlinear Schrödinger equation of the system of the vertically stacked $n$-layers quantum dots demonstrates very good convergent property.

\section{RESULTS AND DISCUSSION}

The physical parameters of InAs/GaAs nanostructures used in our numerical calculation are summarized as follows. For InAs, the energy gap $E_{g}$ is $0.42 \mathrm{eV}, \Delta_{1}$ is $0.38 \mathrm{eV}, m_{1}(0)=0.024 m_{0}$. 


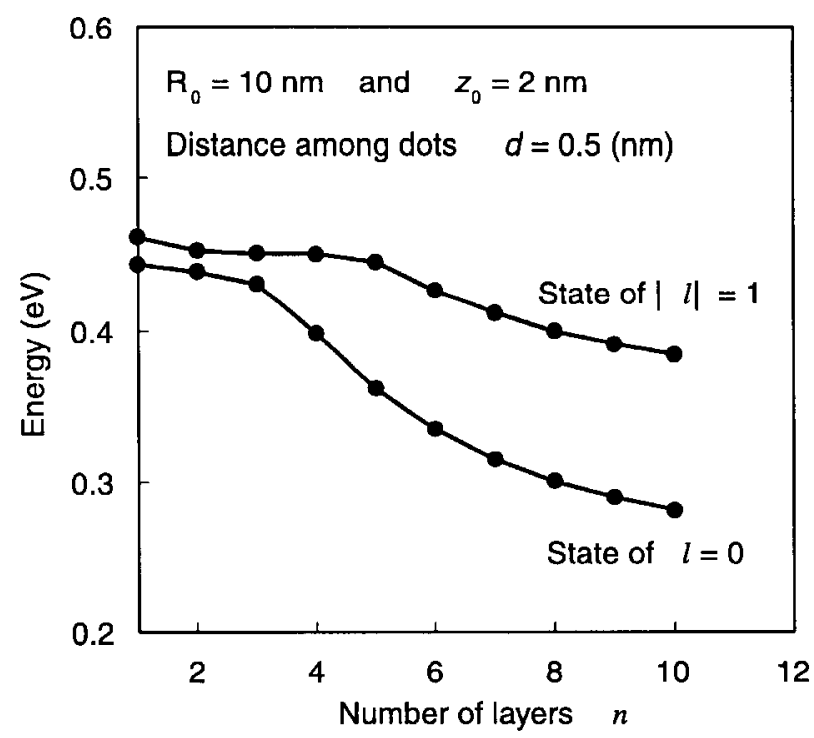

Figure 3. The energy versus the number of stacked QDs.

Table 1. The effects of $d$ on the variation of ground and first excited state energies for the system of the vertically stacked ten-layers QDs.

\begin{tabular}{|c|c|c|c|c|}
\hline Layer's distance $d(\mathrm{~nm})$ & 0.5 & 1.0 & 5.0 & 10.0 \\
\hline Variation of the ground state energy $(l=0)$ & $36 \%$ & $30 \%$ & $18 \%$ & $13 \%$ \\
\hline Variation of the first excited state energy $(|l|=1)$ & $17 \%$ & $14 \%$ & $7 \%$ & $5 \%$ \\
\hline
\end{tabular}

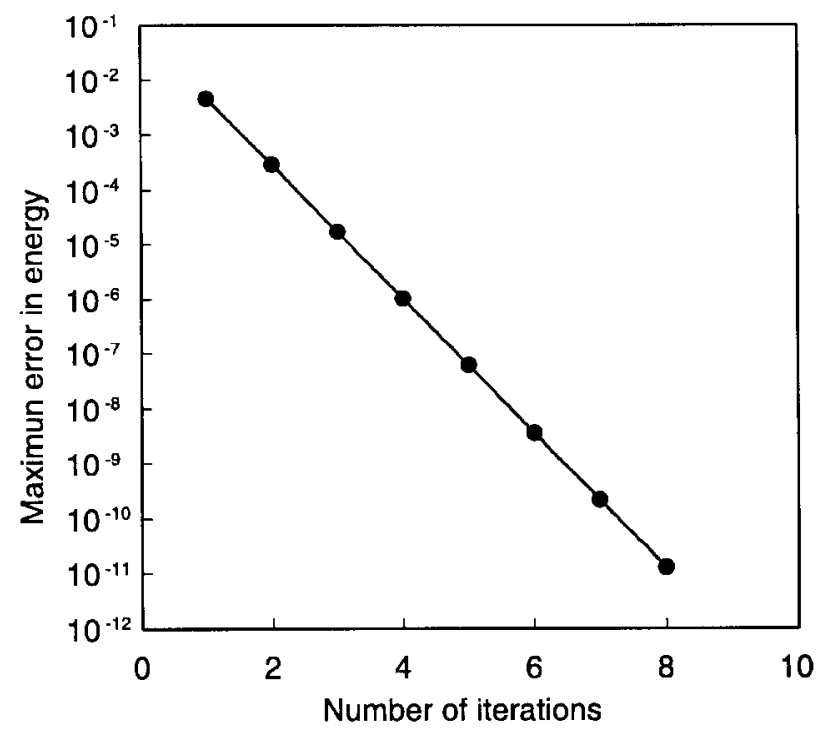

Figure 4. The convergent behavior for a five-layers system.

For $\mathrm{GaAs}$, we set $E_{g}=1.52 \mathrm{eV}, \Delta_{2}=0.34 \mathrm{eV}$, and $m_{2}\left(V_{0}\right)=0.067 m_{0}$. The band offset parameter is taken as $V_{0}=0.55 \mathrm{eV}$. Different $V_{0}$ will result in a small shift in the calculated energy spectra, but we believe that the main tendency should not be changed when the different $V_{0}$ is chosen. As shown in the Figure 3, we first demonstrate the main result of the simulated system of the single and $(n=1)$ vertically stacked $n$-layers InAs/GaAs QDs. We simulate the system of the ten-layers vertically stacked QDs. Each ellipsoidal-shaped QD is separated by distance $d$ among layers. For small dots with $z_{0}=2 \mathrm{~nm}$ and $R_{0}=10 \mathrm{~nm}[13,14]$, and separated by a fixed $d$ we have found 


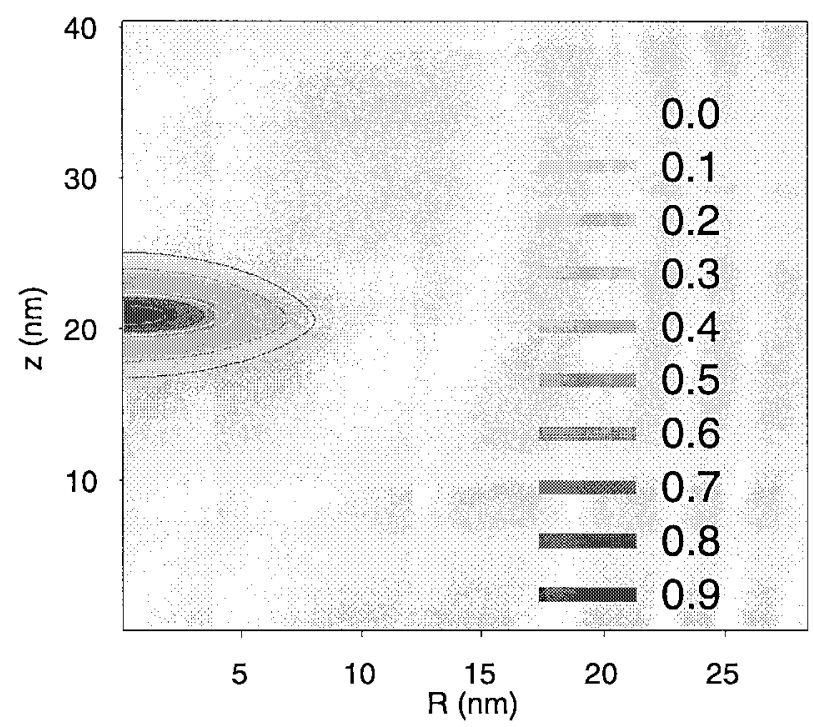

Figure 5. Contour plot of the wave function spreading for the single QD with the state of $l=0$.

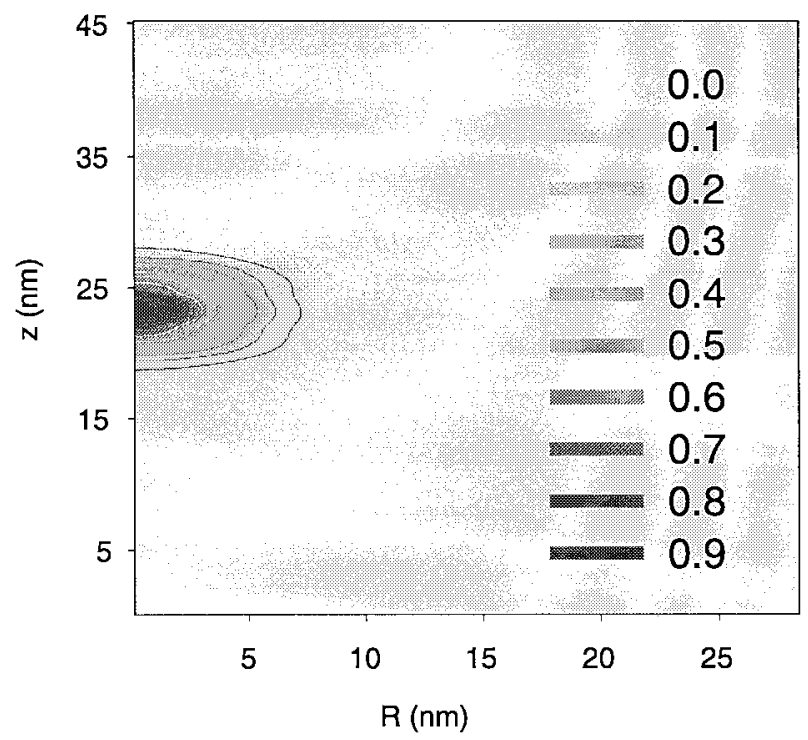

Figure 6. The wave function of the system of the vertically stacked three-layers QDs.

that the transition energy is essentially dominated by the number of stacked $n$-layers QDs. When the stacked number of $n$-layers increases the electron transition energy decreases monotonically and gradually becomes slow when $n>6$. For $d=0.5 \mathrm{~nm}$ the variation of ground state energy can up to $30 \%$. The transition of the first excited state energy $(|l|=1)$ is less dependent on the stacked number of $n$-layers ( $5 \%$ energy variation). It is also found that $d$ plays a crucial role in the tunable states of the dots. For some typical layer's distances, Table 1 summarizes the effects of $d$ on the variation of ground and first excited state energies for the system of the vertically stacked ten-layers QDs.

Figure 4 is the convergence property of the nonlinear iterative method for the system with the vertically stacked five-layers QDs; it takes about eight iteration loops to reach a stopping criterion where the maximum error in energy is less than $10^{-12}$. The global convergence mechanism is due to the effective mass is a monotone function in energy. It takes about 350 seconds to computes all bounded states in a Linux-based PC system, where CPU is Pentium IV $2.5 \mathrm{GHz}$ and RAM is with the 256 MBytes. For other cases, we have similar convergence behavior. In Figures 5 
and 6, contour plots of the normalized wave function for the single and three-layers QDs are reported. Figure 5 is the contour plot of the ground state wave function which confirms the ellipsoidal-shaped QDs. Figure 6 significantly demonstrates the coupling effects and spreading for the system of the vertically coupled three-layers QDs. The computed wave functuion confirms that the coupling effects is significantly dominated by the distance $d$ among the layers of QDs as well as the number of stacked QD layers $n$.

\section{CONCLUSIONS}

A computational effective technique for the single and vertically stacked $n$-layers QDs system simulation has been proposed. With the developed 3-D QDs simulator, we have found that the electron energy spectra are significantly dependent on the number of coupled layers. For $d=0.5 \mathrm{~nm}$, there is about $30 \%$ variation in ground state energy. For the excited states, the layer dependence effect has been found to be weak than that for the ground state. This result is constructive in studying the magneto-optical phenomena and quantum optical structures for the vertically stacked $n$-layers QDs, in particular for optical device applications. The modeling, numerical method, and study presented here not only provide a novel way to simulate the QDs but also are useful to clarify principal dependencies of stacked QDs encrgy states on material band parameter and the number of stacked QDs. We are currently implementing a parallel eigenvalue solver for the simulation of more complicated system of semiconductor quantum dots.

\section{REFERENCES}

1. H. Akinaga and H. Ohno, Semiconductor spintronics, IEEE Transactions on Nanotechnology 1, 19-31, (2002).

2. M. Bayer, P. Hawrylak, K. Hinzer, S. Fafard, M. Korkusinski, Z.R. Wasilewski, O. Stern and A. Forchel, Coupling and entangling of quantum states in quantum dot molecules, Science 291, 451-453, (2001).

3. D. Bimberg, M. Grundmann, F. Ieinrichsdorff, N.N. Ledentsov, V.M. Ustinov, A.E. Zhukov, A.R. Kovsh, M.V. Maximov, Y.M. Shernyakov, B.V. Volovik, A.F. Tsatsul'nikov, P.S. Kop'ev and Zh.I. Alferov, Quantum dot lasers: Breakthrough in optoelectronics, Thin Solid Films 367, 235-249, (2000).

4. A.D. Yoffe, Semiconductor quantum dots and related systems: Electronic, optical, luminescence and related properties of low dimensional systems, Advances in Physics 50, 1-208, (2001).

5. X. Hu and S. Das Sarma, Hilbert-space structure of a solid-state quantum computer: Two-electron states of a double-quantum-dot artificial molecule, Physical Review A 61, 062301-062320, (2000).

6. P. Yu, W. Langbein, K. Leosson and J.M. Hvam, Optical anisotropy in vertically coupled quantum dots, Physical Review B 60, 16680-16685, (1999).

7. Z.J. Zhang, B.W. Li and C.G. Bao, Vertically coupled double quantum dots without tunnelling, Physica $B$ 324, 245-253, (2002)

8. F. Troian, U. Hohenester and E. Molinari, Electron-hole localization in coupled quantum dots, Physical Review B 65, 161301-161304, (2002).

9. Y. Li and H.-M. Lu, Geometric variations and magnetic field effects on electron energy states of InAs/GaAs quantum rings, Japanese Journal of Applied Physics 42, 2404-2407, (2003).

10. Y. Li, O. Voskoboynikov, C.P. Lee, S.M. Sze and O. Tretyak, Electron energy state dependence on the shape and size of semiconductor quantum dots, Journal of Applied Physics 90, 6416-6420, (2001).

11. Y. Li, O. Voskoboynikov, C.-P. Lee and S. M. Sze, A computational technique for electron energy states calculation in nano-scopic three-dimensional InAs/GaAs semiconductor quantum rings simulation, Journal of Computational Electronics 1, 227-230, (2002).

12. Y. Li, Computer simulation of magnetization for 3D ellipsoidal torus-shaped InAs/GaAs quantum rings, International Journal of Modern Physics C: Physics and Computers 14, 501-507, (2003).

13. M. Bayer, G. Ortner, A. Larionov, V. Timofeev, A. Forchel, P. Hawrylak, K. Hinzer, M. Korkusinski, S. Fafardb and Z. Wasilewski, Entangled exciton states in quantum dot molecules, Physica E 12, 900-903, (2002).

14. B. Lita and R.S. Glodman, Nanometer-scale studies of vertical organization and evolution of stacked selfassembled InAs/GaAs quantum dots, Applied Physics Letters 74, 2824-2826, (1999).

15. K. Braman, R. Byers and R. Mathias, The multishift QR algorithm. Part II: Aggressive early deflation, SIAM Journal on Matrix Analysis and Applications 23, 948-973, (2002).

16. D.S. Watkins, QR-like algorithms for eigenvalue problems, Journal of Computational and Applied Mathematics 123, 67-83, (2000).

17. D.C. Sorensen, Implicit application of polynomial filters in a $k$-step Arnoldi method, SIAM Journal on Matrix Analysis and Applications 13, 357-385, (1992).

18. A. Stathopoulos, Y. Saad and K. Wu, Dynamic thick restarting of the Davidson, and the implicitly restarted Arnoldi methods, SIAM Journal on Scientific Computing 19, 227-245, (1998) 
19. R.S. Varga, Matrix Iterative Analysis, Springer, Berlin, (2000).

20. G.H. Golub and C.F. Van Loan, Matrix Computations, The Johns Hopkins University Press, Baltimore, MD, (1996). 